\title{
High-resolution atomic absorption spectrometry combined with machine learning data processing for isotope amount ratio analysis of lithium
}

\author{
Alexander Winckelmann ${ }^{1,2}$, Sascha Nowak ${ }^{3}$, Silke Richter ${ }^{2}$, Sebastian Recknagel ${ }^{2}$, Jens Riedel ${ }^{2}$, Jochen \\ Vogl' ${ }^{2}$, Ulrich Panne ${ }^{1,2}$, Carlos Abad $^{2 *}$ \\ ${ }^{1}$ Humboldt-Universität zu Berlin, Department of Chemistry, Brook-Taylor-Str. 2, 12489 Berlin, Germany \\ ${ }^{2}$ Bundesanstalt für Materialforschung und -prüfung (BAM), Richard-Willstätter-Str. 11, 12489 Berlin, Germany \\ ${ }^{3}$ MEET Battery Research Center, University of Münster, Corrensstr. 46, 48149 Münster, Germany \\ * E-mail corresponding author: carlos.abad@bam.de
}

Section

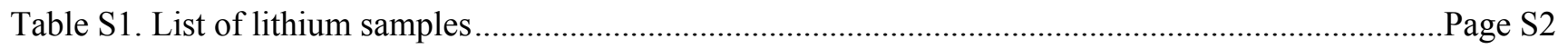

Table S2. Preparation of the chromatographic column with a resin AG-50W-X8 ..................................Page S2

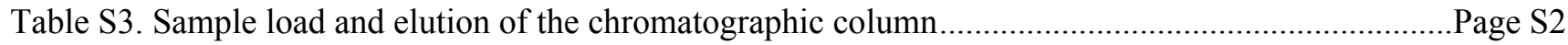

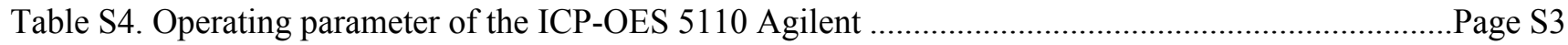

Table S5. Operating parameter of the MC-ICP-MS Neptune Plus ................................................................ S3

Table S6. Temperature program for lithium atomization by HR-CS-GF-AAS …...................................Page S4

Table S7. Isotope amount ratios, isotope amount fractions, isotope delta values, and molar masses of the investigated samples with their associated standard deviation measured by HR-CS-GF-AAS..................Page S4 
Table S1. List of lithium samples

\begin{tabular}{|c|c|c|c|c|c|c|}
\hline Sample & Material & $\begin{array}{l}\text { Sample } \\
\text { preparation }\end{array}$ & $\begin{array}{l}\text { Isotope amount } \\
\text { fraction* } \\
n\left({ }^{6} \mathrm{Li}\right) / n(\mathrm{Li}) \\
\mathrm{mol} \mathrm{mol}^{-1}\end{array}$ & $\boldsymbol{U}$ & $\begin{array}{l}\text { Isotope amount } \\
\text { fraction* } \\
n\left({ }^{7} \mathrm{Li}\right) / n(\mathrm{Li}) \\
\mathrm{mol} \mathrm{mol}^{-1}\end{array}$ & $\boldsymbol{U}$ \\
\hline K 00 & ${ }^{6} \mathrm{Li}$ standard & \multirow{11}{*}{ None } & 0.990482 & 0.000046 & 0.009518 & 0.000046 \\
\hline K 01 & \multirow{9}{*}{$\begin{array}{l}\text { Mixture } \\
{ }^{6} \mathrm{Li}+{ }^{7} \mathrm{Li} \text { standards }\end{array}$} & & 0.90506 & 0.00035 & 0.09494 & 0.00035 \\
\hline K 02 & & & 0.81810 & 0.00042 & 0.18190 & 0.00042 \\
\hline K 03 & & & 0.72964 & 0.00055 & 0.27036 & 0.00055 \\
\hline К 04 & & & 0.63966 & 0.00065 & 0.36034 & 0.00065 \\
\hline K 05 & & & 0.54931 & 0.00070 & 0.45069 & 0.00070 \\
\hline K 06 & & & 0.45577 & 0.00070 & 0.54423 & 0.00070 \\
\hline K 07 & & & 0.36271 & 0.00065 & 0.63729 & 0.00065 \\
\hline K 08 & & & 0.26794 & 0.00055 & 0.73206 & 0.00055 \\
\hline К 09 & & & 0.17001 & 0.00040 & 0.82999 & 0.00040 \\
\hline K 10 & $\begin{array}{l}\mathrm{LiNO}_{3} \\
\text { (Li ICP standard) }\end{array}$ & & 0.07068 & 0.00018 & 0.92932 & 0.00018 \\
\hline A 01 & \multirow{5}{*}{$\begin{array}{l}\text { NMC111 cathode } \\
\text { material }\end{array}$} & \multirow{5}{*}{$\begin{array}{l}\text { Dissolution in } \\
\mathrm{HNO}_{3} / \mathrm{Cl}(3: 1), \\
\text { ion-exchange } \\
\text { chromatography }\end{array}$} & 0.07491 & 0.00020 & 0.92509 & 0.00020 \\
\hline A 02 & & & 0.07490 & 0.00020 & 0.92510 & 0.00020 \\
\hline A 03 & & & 0.07491 & 0.00020 & 0.92509 & 0.00020 \\
\hline A 04 & & & 0.07490 & 0.00020 & 0.92510 & 0.00020 \\
\hline A 05 & & & 0.07490 & 0.00020 & 0.92510 & 0.00020 \\
\hline A 06 & $\mathrm{Li}_{2} \mathrm{CO}_{3}$ & \multirow{4}{*}{ None } & 0.07610 & 0.00020 & 0.92390 & 0.00020 \\
\hline A 07 & $\mathrm{Li}_{2} \mathrm{CO}_{3}$ & & 0.07485 & 0.00020 & 0.92515 & 0.00020 \\
\hline A 08 & $\mathrm{LiCl}$ & & 0.07555 & 0.00020 & 0.92445 & 0.00020 \\
\hline A 09 & $\mathrm{LiOH}$ & & 0.07586 & 0.00021 & 0.92414 & 0.00021 \\
\hline A 10 & $\mathrm{LiF}$ & $\begin{array}{l}\text { Ion-exchange } \\
\text { chromatography }\end{array}$ & 0.05966 & 0.00016 & 0.94034 & 0.00016 \\
\hline LSVEC & $\mathrm{Li}_{2} \mathrm{CO}_{3}$ & \multirow{2}{*}{ None } & 0.07591 & 0.00021 & 0.92409 & 0.00021 \\
\hline IRMM-016 & $\mathrm{Li}_{2} \mathrm{CO}_{3}$ & & 0.075889 & 0.000075 & 0.924111 & 0.000075 \\
\hline
\end{tabular}

* Values from measurements using MC-ICP-MS (experimental section), except for the certified reference materials LSVEC and IRMM016, which were taken from the certificates of analysis.

Table S2. Preparation of the chromatographic column with a resin AG-50W-X8

\begin{tabular}{lll}
\hline Step & Solution & Volume \\
\hline Rinse & $\mathrm{H}_{2} \mathrm{O}$ & 5 column volume $(15 \mathrm{~mL})$ \\
Load resin & $\mathrm{AG} 50 \mathrm{~W}-\mathrm{X} 8$ in $\mathrm{H}_{2} \mathrm{O}$ & $3 \mathrm{~mL}$ \\
Clean resin & $6 \mathrm{~mol} \mathrm{~L}-1 \mathrm{HCl}$ & 4 column volume $(12 \mathrm{~mL})$ \\
Backwash & $\mathrm{H}_{2} \mathrm{O}$ & 1 column volume $(3 \mathrm{~mL})$ \\
Conditioning & $0.5 \mathrm{~mol} \mathrm{~L}^{-1} \mathrm{HCl}$ & 5 column volume $(15 \mathrm{~mL})$ \\
\hline
\end{tabular}

Table S3. Sample load and elution of the chromatographic column

\begin{tabular}{lll}
\hline Step & Solution & Volume \\
\hline Load sample & Lithium solution & $2 \mathrm{~mL}$ \\
Elution & $0.5 \mathrm{~mol} \mathrm{~L}^{-1} \mathrm{HCl}$ & $30 \mathrm{~mL}$ \\
Clean resin & $6 \mathrm{~mol} \mathrm{~L}^{-1} \mathrm{HCl}$ & 4 column volume $(12 \mathrm{~mL})$ \\
Backwash & $\mathrm{H}_{2} 0$ & 1 column volume $(3 \mathrm{~mL})$ \\
Conditioning & $0.5 \mathrm{~mol} \mathrm{~L}^{-1} \mathrm{HCl}$ & 5 column volume $(15 \mathrm{~mL})$ \\
\hline
\end{tabular}


Table S4. Operating parameter of the ICP-OES 5110 Agilent

\begin{tabular}{ll}
\hline Parameter & Value \\
\hline Forward power/ W & 1200 \\
Plasma gas flow rate / $\mathrm{L} \mathrm{min}^{-1}$ & 12 \\
Auxiliary gas flow rate / $\mathrm{L} \mathrm{min}^{-1}$ & 1 \\
Make up gas / L min & 0 \\
Carrier gas flow rate / $\mathrm{L} \mathrm{min}^{-1}$ & 0.7 \\
Peri-pump speed / $\mathrm{min}^{-1}$ & 10 \\
Analysis time / s & 10 \\
Sample feed time / s & 45 \\
Rinse time / s & 30 \\
\hline
\end{tabular}

Table S5. Operating parameter of the MC-ICP-MS Neptune Plus

\begin{tabular}{ll}
\hline Parameter & Value \\
\hline Instrument type & Neptune Plus \\
Autosampler & ESI SC2 DX \\
Aspiration mode & Self-aspirating \\
Nebulizer & PFA $100 \mu \mathrm{L} \mathrm{min}{ }^{-1}$ \\
Spray chamber & Double-Scott-Cyclonic (SIS) \\
Interface & Jet \\
Cones & Ni sampler \& Ni H skimmer \\
Cool gas flow rate in $\mathrm{L} \mathrm{min}^{-1}$ & $\approx 16$ \\
Auxiliary gas flow rate in $\mathrm{L} \mathrm{min}^{-1}$ & $\approx 0.9$ \\
Sample gas flow rate in $\mathrm{L} \mathrm{min}^{-1}$ & $1.08-1.10$ \\
RF power in W & 1200 \\
Guard electrode & On \\
Mass resolution mode & Low \\
Faraday detectors & L4, H4 \\
Gain calibration & at least once a month \\
Baseline measurement & at least once a month \\
Peak center ${ }^{a}$ & each measurement \\
Amplifier resistors in $\Omega$ & $10^{11}$ \\
Integration time in s & 4.194 \\
Blocks / cycles & $1 / 50$ \\
Sensitivity in V $\cdot \mathrm{mg}^{-1} \cdot \mathrm{kg}^{b}$ & 22 to 25 \\
Li mass fractions of solutions used in $\mathrm{mg} \mathrm{kg}^{-1}$ & 0.5 \\
Typical ${ }^{7} \mathrm{Li}$ blank intensity in $\mathrm{mV}^{c}$ & 35 to 130 \\
Typical isotope ratio drift in $\%{ }^{d}$ & 0.1 to 0.2 \\
Typical isotope ratios repeatability $\left(s_{\mathrm{rel}}\right)$ in $\%{ }^{e}$ & $\approx 0.1$ \\
Isotope ratio repeatability $\left(s_{\mathrm{rel}}, \mathrm{n}\right)$ in $\%{ }^{f}$ & $0.42, n=31$ \\
\hline
\end{tabular}

a Peak center before each sample and standard measurement

${ }^{b}$ Sum of all $\mathrm{Li}$ ion intensities per $1 \mathrm{mg} \mathrm{kg}^{-1} \mathrm{Li}$ in the solution

c Blank intensity strongly increased within a measurement sequence from 35 to $130 \mathrm{mV}$ due to memory effects

$d$ Typical drift for the ${ }^{7} \mathrm{Li} /{ }^{6} \mathrm{Li}$ ratio between two consecutive standard measurements (median of all absolute drift values)

$e$ Relative standard deviation $s_{\text {rel }}$ within one measurement $\left({ }^{7} \mathrm{Li} /{ }^{\circ} \mathrm{Li}, \mathrm{LSVEC}\right)$

$f$ Relative standard deviation $s_{\text {rel }}$ of $n$ repeated measurements $\left({ }^{7} \mathrm{Li} /{ }^{6} \mathrm{Li}, \mathrm{LSVEC}\right)$, example for low drift effects; in case of strong drift effects this value can go up to a value of a few per mil 
Table S6. Temperature program for lithium atomization by HR-CS-GF-AAS

\begin{tabular}{|c|c|c|c|c|c|c|c|}
\hline \multirow[t]{2}{*}{ Step } & \multirow[t]{2}{*}{ Name } & \multirow{2}{*}{$\begin{array}{l}\text { Temp } \\
/{ }^{\circ} \mathrm{C}\end{array}$} & \multirow{2}{*}{$\begin{array}{l}\text { Ramp } \\
/{ }^{\circ} \mathrm{C} \mathrm{s}^{-1}\end{array}$} & \multirow{2}{*}{$\begin{array}{l}\text { Hold } \\
\text { / s }\end{array}$} & \multirow{2}{*}{$\begin{array}{l}\text { Time } \\
/ \mathrm{s}\end{array}$} & \multicolumn{2}{|c|}{ Gas flow } \\
\hline & & & & & & $\begin{array}{l}\text { Primary } \\
\text { / L } \text { min }^{-1}\end{array}$ & $\begin{array}{l}\text { Secondary } \\
\text { / L } \mathbf{~ m i n}^{-1}\end{array}$ \\
\hline 1 & Dry & 70 & 6 & 15 & 22.5 & 2.0 & - \\
\hline 2 & Dry & 80 & 6 & 20 & 21.7 & 2.0 & - \\
\hline 3 & Dry & 90 & 3 & 20 & 23.3 & 2.0 & - \\
\hline 4 & Dry & 110 & 5 & 10 & 14.0 & 2.0 & - \\
\hline 5 & Pyrolysis 1 & 350 & 50 & 20 & 24.8 & 2.0 & - \\
\hline 6 & Pyrolysis 2 & 750 & 300 & 10 & 11.3 & 0.1 & - \\
\hline 7 & Gas adaptation & 750 & 0 & 5 & 5.0 & - & - \\
\hline 8 & Atomization & 2350 & 1500 & 10 & 11.0 & - & - \\
\hline 9 & Clean out 1 & 2450 & 500 & 10 & 10.2 & - & 0.5 \\
\hline 10 & Clean out 2 & 2600 & 500 & 4 & 4.3 & 2.0 & - \\
\hline
\end{tabular}

Table S7. Isotope amount ratios, isotope amount fractions, isotope delta values, and molar masses of the investigated samples with their associated standard deviation measured by HR-CS-GF-AAS

\begin{tabular}{|c|c|c|c|c|c|c|c|c|c|}
\hline Sample & \multicolumn{3}{|c|}{$\begin{array}{l}\text { Isotope amount fraction } \\
\text { / } \mathrm{mol} \mathrm{mol}^{-1}\end{array}$} & \multicolumn{2}{|c|}{$\begin{array}{l}\text { Isotope amount ratio } \\
/ \mathrm{mol} \mathrm{mol}^{-1}\end{array}$} & \multicolumn{2}{|l|}{$\begin{array}{l}\text { Delta } \\
\text { / \%o }\end{array}$} & \multicolumn{2}{|c|}{ Molar mass } \\
\hline A 1 & 0.07499 & 0.92501 & 0.00012 & 12.336 & 0.021 & 12.2 & 1.7 & 6.94095 & 0.00012 \\
\hline A 2 & 0.07490 & 0.92510 & 0.00021 & 12.351 & 0.038 & 13.4 & 3.1 & 6.94104 & 0.00021 \\
\hline A 3 & 0.07498 & 0.92502 & 0.00010 & 12.336 & 0.018 & 12.2 & 1.5 & 6.94095 & 0.00010 \\
\hline A 4 & 0.07498 & 0.92502 & 0.00008 & 12.336 & 0.015 & 12.2 & 1.2 & 6.94095 & 0.00008 \\
\hline A 5 & 0.07490 & 0.92510 & 0.00010 & 12.351 & 0.017 & 13.4 & 1.4 & 6.94103 & 0.00010 \\
\hline A 1 (without IC) & 0.07493 & 0.92507 & 0.00009 & 12.346 & 0.016 & 13.0 & 1.3 & 6.94101 & 0.00009 \\
\hline A 2 (without IC) & 0.07492 & 0.92508 & 0.00007 & 12.347 & 0.012 & 13.1 & 1.0 & 6.94102 & 0.00007 \\
\hline A 3 (without IC) & 0.07493 & 0.92507 & 0.00007 & 12.346 & 0.013 & 13.0 & 1.1 & 6.94101 & 0.00007 \\
\hline A 4 (without IC) & 0.07492 & 0.92508 & 0.00007 & 12.347 & 0.013 & 13.1 & 1.0 & 6.94102 & 0.00007 \\
\hline A 5 (without IC) & 0.07490 & 0.92510 & 0.00008 & 12.352 & 0.014 & 13.5 & 1.1 & 6.94104 & 0.00008 \\
\hline A 6 & 0.07592 & 0.92408 & 0.00013 & 12.172 & 0.022 & -1.3 & 1.8 & 6.94002 & 0.00013 \\
\hline A 7 & 0.07499 & 0.92501 & 0.00009 & 12.336 & 0.016 & 12.2 & 1.4 & 6.94095 & 0.00009 \\
\hline A 8 & 0.07550 & 0.92450 & 0.00013 & 12.246 & 0.022 & 4.8 & 1.8 & 6.94044 & 0.00013 \\
\hline A 9 & 0.07582 & 0.92418 & 0.00014 & 12.190 & 0.024 & 0.2 & 1.9 & 6.94012 & 0.00014 \\
\hline LSVEC & 0.07583 & 0.92417 & 0.00013 & 12.187 & 0.023 & & & 6.94011 & 0.00013 \\
\hline IRMM-016 & 0.07578 & 0.92422 & 0.00012 & 12.196 & 0.022 & 0.7 & 1.8 & 6.94015 & 0.00012 \\
\hline
\end{tabular}

